\title{
Efeito do alumínio na absorção e na utilização de macronutrientes em duas cultivares de arroz ${ }^{(1)}$
}

\author{
Renilton Joaquim de Mendonça( ${ }^{(2)}$, José Cambraia ${ }^{(3)}$, Juraci Alves de Oliveira( ${ }^{(3)}$ \\ e Marco Antonio Oliva(4)
}

\begin{abstract}
Resumo - Para avaliar o efeito do Al na absorção e na utilização de alguns macronutrientes, as cultivares de arroz Fernandes e Maravilha, respectivamente, tolerante e sensível a esse metal, foram expostas ao Al, em solução nutritiva, durante 20 dias, quando determinaram-se os teores, a absorção líquida e a eficiência de utilização de $\mathrm{P}, \mathrm{K}, \mathrm{Ca}$ e magnésio. A absorção líquida e os teores desses macronutrientes na presença de $\mathrm{Al}$ decresceram na parte aérea e nas raízes das duas cultivares, especialmente na concentração mais elevada de $\mathrm{Al}$ e na cultivar sensível. Os macronutrientes que tiveram os teores mais reduzidos pelo $\mathrm{Al}$ nas raízes foram $\mathrm{K}>\mathrm{Mg}>\mathrm{Pe} \mathrm{Ca}>\mathrm{Mg}=\mathrm{K}>\mathrm{Pe}$ na parte aérea, $\mathrm{Mg}>\mathrm{Ca}>\mathrm{Pe} \mathrm{Mg}>\mathrm{Ca}>\mathrm{P}>\mathrm{K}$, nas cultivares tolerante e sensível, respectivamente. Os macronutrientes que tiveram a absorção mais reduzida pelo $\mathrm{Al}$ foram $\mathrm{Mg}>\mathrm{Ca}>\mathrm{P}>\mathrm{K}$, nas duas cultivares. Na presença de $\mathrm{Al}$, a cultivar tolerante apresentou eficiência de utilização mais elevada de todos os macronutrientes. Os índices de utilização de $\mathrm{P}, \mathrm{Ca}$ e $\mathrm{Mg}$ aumentaram na cultivar tolerante, mas decresceram em termos de $\mathrm{P}$ e $\mathrm{K}$, aumentando apenas para $\mathrm{Mg}$, na cultivar sensível. A tolerância diferencial dessas cultivares de arroz ao Al resulta, provavelmente, de suas diferenças em absorver, manter concentrações adequadas e utilizar com maior eficiência esses macronutrientes, principalmente $\mathrm{Mg}, \mathrm{Ca}$ e fósforo.
\end{abstract}

Termos para indexação: Oryza sativa, nutrientes minerais, absorção de nutriente, toxicidade, tolerância.

\section{Aluminum effects on the uptake and utilization of macronutrients in two rice cultivars}

Abstract - To evaluate the effect of $\mathrm{Al}$ on the uptake and utilization of some macronutrients, the rice cultivars Fernandes and Maravilha, respectively tolerant and sensitive to this metal, were exposed to $\mathrm{Al}$ in nutrient solution for 20 days, and the contents, uptake and efficiency of utilization of $\mathrm{P}, \mathrm{K}, \mathrm{Ca}$ and $\mathrm{Mg}$ were determined. The uptake and the contents of these macronutrients decreased in the presence of $\mathrm{Al}$ in the aerial parts and roots of both cultivars, especially at the higher $\mathrm{Al}$ concentration and in the $\mathrm{Al}$ sensitive cultivar. The macronutrient contents more reduced by $\mathrm{Al}$ in the roots were $\mathrm{K}>\mathrm{Mg}>\mathrm{P}$ and $\mathrm{Ca}>\mathrm{Mg}=\mathrm{K}>\mathrm{P}$ and in the aerial parts were $\mathrm{Mg}>\mathrm{Ca}>\mathrm{P}$ and $\mathrm{Mg}>\mathrm{Ca}>\mathrm{P}>\mathrm{K}$ in the tolerant and sensitive cultivars, respectively. The macronutrients uptake more reduced by $\mathrm{Al}$ were $\mathrm{Mg}>\mathrm{Ca}>\mathrm{P}>\mathrm{K}$ in both cultivars. In the presence of $\mathrm{Al}$, the tolerant cultivar showed higher efficiency of utilization of all studied macronutrients. The utilization indices of $\mathrm{P}, \mathrm{Ca}$ and $\mathrm{Mg}$ increased in the tolerant cultivar, but decreased for $\mathrm{P}$ and $\mathrm{K}$, increasing only for $\mathrm{Mg}$ in the sensitive cultivar. The results suggest that the differential tolerance of these rice cultivars to $\mathrm{Al}$ may be due to their differences in uptake, ability to keep adequate concentrations and to use these macronutrients with higher efficiency, especially $\mathrm{Mg}$, $\mathrm{Ca}$ and phosphorus.

Index terms: Oryza sativa, mineral nutrients, nutrient uptake, toxicity, tolerance.

(1) Aceito para publicação em 29 de abril de 2003.

Extraído da tese de mestrado apresentada pelo primeiro autor à Universidade Federal de Viçosa (UFV), Viçosa, MG.

${ }^{(2)}$ Rua 05, Quadra 06, Lote 15, Setor do Lago, CEP 76550-000 Porangatu, GO. E-mail: rjmendoca@yahoo.com.br

(3) UFV, Dep. de Biologia Geral, Avenida P. H. Rolfs s/no CEP 36571-000 Viçosa, MG. Bolsista do CNPq. E-mail: cambraia@ufv.br

(4) UFV, Dep. de Biologia Vegetal. Bolsista do CNPq. E-mail: oliva@ufv.br

\section{Introdução}

O arroz é uma cultura capaz de se adaptar a diferentes tipos de ambiente, incluindo solos de baixa fertilidade ou com problema de toxidez de alumínio. Existe entre as cultivares de arroz ampla variação quanto à tolerância ao Al (Fageria et al., 1988; Sivaguru et al., 1992). E entre as culturas de importância econômica, o arroz é uma das mais tolerantes 
a este elemento (Fageria et al., 1988; Ferreira et al., 1995).

A tolerância de várias espécies vegetais ao $\mathrm{Al}$ tem sido atribuída à capacidade de as plantas manterem em suas raízes ou na parte aérea níveis adequados de certos macronutrientes e micronutrientes essenciais (Rengel \& Robinson, 1989a, 1989b; Tan et al., 1993; Malkanthi et al., 1995b). Em arroz, este tipo de correlação tem sido testado em diferentes cultivares, tanto no Brasil (Fageria \& Carvalho, 1982; Fageria et al., 1988) como no exterior (Sivaguru \& Paliwal, 1993; Jan \& Pettersson, 1995). As cultivares tolerantes de arroz apresentam, quase sempre, teores de $\mathrm{P}$ e de Ca mais elevados do que as cultivares sensíveis (Fageria, 1985; Sivaguru \& Paliwal, 1993). Este tipo de correlação, segundo Furlani \& Furlani (1991), entretanto, pode ser apenas a resultante de maior crescimento e aprofundamento dos sistemas radiculares das variedades tolerantes, que explorariam maior volume de solo, e não necessariamente em razão de um aumento na eficiência de absorção ou uso desses minerais. Outros estudos indicam que, além da capacidade de manter teores mais elevados de certos minerais, algumas cultivares de arroz tolerantes ao $\mathrm{Al}$ apresentam maior eficiência na absorção e na utilização desses elementos, sob condições de estresse (Sivaguru \& Paliwal, 1993). Embora já sejam conhecidas entre as cultivares de arroz comercialmente importantes as mais tolerantes ao Al, os mecanismos e os principais fatores que afetam essa tolerância, como acontece com outras espécies, ainda não foram esclarecidos.

O objetivo deste trabalho foi avaliar o efeito do Al na absorção e utilização de macronutrientes em duas cultivares de arroz com tolerância diferencial ao alumínio.

\section{Material e Métodos}

Foram utilizadas no presente trabalho sementes de duas cultivares de arroz (Oryza sativa L.) fornecidas pela Embrapa-Centro Nacional de Pesquisa de Arroz e Feijão (CNPAF), uma tolerante (Fernandes: CNA-1158) e outra sensível (Maravilha: CNA-6843-1) ao Al (Fageria \& Carvalho, 1982; Fageria et al., 1988).

As sementes, selecionadas quanto ao tamanho e forma, foram tratadas com ácido sulfúrico $\left(\mathrm{H}_{2} \mathrm{SO}_{4}\right) 50 \%$ por 15 minutos, lavadas em água de torneira, esterilizadas superficialmente, por tratamento com hipoclorito de sódio $2 \%$ por 15 minutos e lavadas, novamente, em água corrente e em água desmineralizada. A seguir, foram colocadas para germinar em cartuchos de papel "germtest" ( $\mathrm{pH}$ neutro), mergulhados em vasos de plástico contendo 1,6 L de solução nutritiva de Clark (Clark, 1975), pH 4,0, com um quinto de força iônica, sob aeração contínua.

As plântulas, após seleção quanto à uniformidade de forma e tamanho, foram transplantadas para vasos de polietileno pintados externamente de preto contendo 1,6 L de solução nutritiva de Clark, pH 4,0 (Clark, 1975), modificada para conter as seguintes concentrações de Al: 0 , 0,75 e $1,50 \mathrm{mM}$, sob a forma de $\mathrm{Al}_{2}\left(\mathrm{SO}_{4}\right)_{3} \cdot 18 \mathrm{H}_{2} \mathrm{O} . \mathrm{O} \mathrm{pH}$ da solução nutritiva foi ajustado diariamente para 4,0 pela adição de $\mathrm{HCl} 0,1 \mathrm{~N}$ ou $\mathrm{NaOH} 0,1 \mathrm{~N}$ e as soluções nutritivas renovadas a cada sete dias. No momento da aplicação dos tratamentos com $\mathrm{Al}$, quando as plântulas estavam com 10 dias de idade foram retiradas amostras de tecidos das duas cultivares para as determinações da matéria seca e dos teores dos macronutrientes: $\mathrm{P}, \mathrm{K}, \mathrm{Ca}$ e $\mathrm{Mg}\left(\mathrm{T}_{1}=\right.$ tempo zero, início da exposição ao $\mathrm{Al}$ ), necessários ao cálculo das taxas de absorção líquida (Welbank, 1962). Após 20 dias de exposição ao Al, as plantas foram colhidas, lavadas em água corrente e enxaguadas em água desmineralizada. Determinou-se, então, a massa de matéria seca das duas partes da planta após serem secadas em estufa a $70^{\circ} \mathrm{C}$, até massa constante. Após mineralização das amostras com uma mistura nítrico-perclórica, os teores de $\mathrm{P}$ foram determinados pelo método de Lindeman (1958) e os de K, Ca e Mg por espectrofotometria de absorção atômica.

A obtenção das plântulas e os experimentos foram todos realizados em sala de crescimento com temperatura controlada $\left(25 \pm 1^{\circ} \mathrm{C}\right)$, fluxo de fótons fotossintéticos de $230 \mu \mathrm{mol} \mathrm{m} \mathrm{m}^{-2} \mathrm{~s}^{-1} \mathrm{e}$ fotoperíodo de 16 horas.

A taxa de absorção líquida dos elementos minerais por unidade de massa de matéria seca de raízes foi estimada pela fórmula proposta por Welbank (1962):

Taxa de absorção líquida $=\left[\frac{\left(\mathrm{M}_{2}-\mathrm{M}_{1}\right)}{\left(\mathrm{T}_{2}-\mathrm{T}_{1}\right)}\right]\left[\frac{\left(\ln \mathrm{W}_{2}-\ln \mathrm{W}_{1}\right)}{\left(\mathrm{W}_{2}-\mathrm{W}_{1}\right)}\right]$,

em que: $\mathrm{M}_{2}$ e $\mathrm{M}_{1}$ é o conteúdo total do nutriente encontrado na parte aérea e no sistema radicular nos tempos $\mathrm{T}_{2}$ (fim do experimento) e $\mathrm{T}_{1}$ (início da exposição ao $\mathrm{Al}$ ), respectivamente; $\mathrm{W}_{2}$ e $\mathrm{W}_{1}$ é a massa de matéria seca das raízes, nos tempos $\mathrm{T}_{2}$ e $\mathrm{T}_{1}$, respectivamente.

A eficiência de utilização (EU) dos elementos minerais foi avaliada por meio do índice de eficiência de utilização, conforme Siddiqi \& Glass (1981): 
Índice de eficiência de utilização $=\frac{(\mathrm{W})^{2}}{\mathrm{M}}$,

em que: W é a massa de matéria seca total da planta; $M$ é o conteúdo total do nutriente na planta.

O experimento foi disposto segundo esquema fatorial, no delineamento inteiramente casualizado, com três repetições. Todos os resultados, expressos em função da massa de matéria seca, foram submetidos à análise de variância e as médias comparadas pelo teste de Tukey, a 5\% de probabilidade.

\section{Resultados e Discussão}

Os teores dos elementos minerais, de modo geral, decresceram nas raízes das duas cultivares com o aumento na concentração de Al na solução nutritiva (Tabela 1). Na concentração de $0,75 \mathrm{mM}$ de Al, observou-se, na cultivar tolerante, decréscimo apenas no teor de $\mathrm{Mg}$, enquanto na sensível o decréscimo ocorreu nos teores de K e magnésio. Nesta concentração de $\mathrm{Al}$, o teor de $\mathrm{P}$ aumentou na cultivar tolerante, mas permaneceu inalterado na sensível. Aumentos nos teores de $\mathrm{P}$, como o verificado na cultivar tolerante, têm sido observados também em outras espécies (Calbo \& Cambraia, 1980; Tan et al., 1993; Malkanthi et al., 1995b). Na concentração mais elevada de $\mathrm{Al}$, observaram-se decréscimos nos teores dos elementos minerais nas duas cultivares, com exceção do $\mathrm{Ca}$, na cultivar tolerante.
Os decréscimos observados nesta concentração de $\mathrm{Al}$, entretanto, se intensificaram em relação à concentração de $0,75 \mathrm{mM}$ apenas em $\mathrm{P}$ e $\mathrm{K}$ na cultivar tolerante, mas em $\mathrm{P}, \mathrm{K}$ e $\mathrm{Ca}$ na cultivar sensível. As cultivares não diferiram entre si quanto ao teor de $\mathrm{P}$, independentemente da concentração de $\mathrm{Al}$ na solução nutritiva. Na ausência de $\mathrm{Al}$, a cultivar sensível apresentou teor mais elevado de $\mathrm{K}$ do que a cultivar tolerante, embora não se tenha observado diferenças significativas quanto aos teores de $\mathrm{Ca}$ e magnésio. Na presença de $\mathrm{Al}$, a cultivar tolerante apresentou teores mais elevados de $\mathrm{K}, \mathrm{Ca}$ e $\mathrm{Mg}$ do que a cultivar sensível, especialmente na concentração mais elevada de alumínio. Os macronutrientes que mais sofreram os efeitos tóxicos do Al foram, pela ordem: $\mathrm{K}>\mathrm{Mg}>\mathrm{P}$ na tolerante e $\mathrm{Ca}>\mathrm{Mg}=\mathrm{K}>\mathrm{P}$ na sensível. Quanto ao $\mathrm{Ca}$, enquanto a cultivar sensível sofreu redução de $73 \%$ no teor deste elemento na concentração mais elevada de $\mathrm{Al}$, a tolerante não sofreu nenhuma mudança significativa. Parte da tolerância diferencial ao $\mathrm{Al}$ dessas cultivares, portanto, pode estar associada à capacidade de a cultivar tolerante manter adequado o teor de $\mathrm{Ca}$ em suas raízes.

Os teores de $\mathrm{P}, \mathrm{K}, \mathrm{Ca}$ e $\mathrm{Mg}$ na parte aérea, de modo geral, também sofreram reduções nas duas cultivares, principalmente na concentração mais elevada de alumínio (Tabela 1). Os teores de $\mathrm{P}$ e $\mathrm{K}$ fo-

Tabela 1. Efeito do Al sobre os teores, a absorção líquida e a eficiência de utilização de P, K, Ca e Mg nas cultivares de arroz Fernandes e Maravilha, respectivamente, tolerante e sensível ao alumínio ${ }^{(1)}$.

\begin{tabular}{|c|c|c|c|c|c|c|c|c|}
\hline \multirow[t]{2}{*}{$\mathrm{Al}(\mathrm{mM})$} & \multicolumn{4}{|c|}{ Cultivar tolerante } & \multicolumn{4}{|c|}{ Cultivar sensível } \\
\hline & $P$ & $\mathrm{~K}$ & $\mathrm{Ca}$ & $\mathrm{Mg}$ & $\mathrm{P}$ & $\mathrm{K}$ & $\mathrm{Ca}$ & $\mathrm{Mg}$ \\
\hline & \multicolumn{8}{|c|}{ Teor dos nutrientes nas raízes $\left(\mathrm{mg} \mathrm{g}^{-1}\right)$} \\
\hline 0,00 & $7,6 \mathrm{Ba}$ & $15,2 \mathrm{Ab}$ & $1,3 \mathrm{Aa}$ & $1,3 \mathrm{Aa}$ & $8,3 \mathrm{Aa}$ & $18,1 \mathrm{Aa}$ & $1,1 \mathrm{Aa}$ & $1,1 \mathrm{Aa}$ \\
\hline 0,75 & $8,8 \mathrm{Aa}$ & $15,4 \mathrm{Aa}$ & $1,1 \mathrm{Aa}$ & $1,0 \mathrm{Ba}$ & $8,2 \mathrm{Aa}$ & $14,0 \mathrm{Bb}$ & $0,9 \mathrm{Aa}$ & $0,5 \mathrm{Bb}$ \\
\hline 1,50 & $6,5 \mathrm{Ca}$ & $7,1 \mathrm{Ba}$ & $1,1 \mathrm{Aa}$ & $0,9 \mathrm{Ba}$ & $5,8 \mathrm{Ba}$ & $6,4 \mathrm{Cb}$ & $0,3 \mathrm{Bb}$ & $0,4 \mathrm{Bb}$ \\
\hline & \multicolumn{8}{|c|}{ Teor dos nutrientes na parte aérea $\left(\mathrm{mg} \mathrm{g}^{-1}\right)$} \\
\hline 0,00 & $10,0 \mathrm{Aa}$ & $27,6 \mathrm{Ba}$ & $2,5 \mathrm{Aa}$ & $4,6 \mathrm{Aa}$ & $9,0 \mathrm{Ab}$ & $21,1 \mathrm{Ab}$ & $2,2 \mathrm{Ab}$ & $4,8 \mathrm{Aa}$ \\
\hline 0,75 & $10,3 \mathrm{Aa}$ & $30,0 \mathrm{Aa}$ & $2,6 \mathrm{Aa}$ & $3,0 \mathrm{Ba}$ & $7,4 \mathrm{Bb}$ & $20,3 \mathrm{Ab}$ & $1,2 \mathrm{Bb}$ & $3,0 \mathrm{Ba}$ \\
\hline 1,50 & $5,3 \mathrm{Ba}$ & $27,6 \mathrm{Ba}$ & $1,2 \mathrm{Ba}$ & $2,1 \mathrm{Ca}$ & $4,4 \mathrm{Cb}$ & $16,2 \mathrm{Bb}$ & $1,0 \mathrm{Ba}$ & $1,4 \mathrm{Cb}$ \\
\hline \multicolumn{9}{|c|}{ Absorção líquida dos nutrientes $\left(\mu \mathrm{mol} \mathrm{g}^{-1} \operatorname{dia}^{-1}\right)$} \\
\hline 0,00 & $253 \mathrm{Aa}$ & $525 \mathrm{Aa}$ & $44 \mathrm{Aa}$ & $132 \mathrm{Aa}$ & $199 \mathrm{Ab}$ & $359 \mathrm{Ab}$ & $32 \mathrm{Ab}$ & $115 \mathrm{Ab}$ \\
\hline 0,75 & $194 \mathrm{Ba}$ & 399Ba & $31 \mathrm{Ba}$ & $60 \mathrm{Ba}$ & $134 \mathrm{Bb}$ & $260 \mathrm{Bb}$ & $13 \mathrm{Bb}$ & $53 \mathrm{Ba}$ \\
\hline 1,50 & $112 \mathrm{Ca}$ & $360 \mathrm{Ca}$ & $16 \mathrm{Ca}$ & $46 \mathrm{Ca}$ & $74 \mathrm{Cb}$ & $172 \mathrm{Cb}$ & $8 \mathrm{Cb}$ & $20 \mathrm{Cb}$ \\
\hline \multicolumn{9}{|c|}{ Eficiência de utilização dos nutrientes $\left(\mathrm{g}^{2} \mathrm{mmol}^{-1}\right)$} \\
\hline 0,00 & $3,2 \mathrm{Ba}$ & $1,5 \mathrm{Aa}$ & $17,4 \mathrm{Ba}$ & $6,0 \mathrm{Ba}$ & 2,9Aa & 1,6Аa & $16,6 \mathrm{Aa}$ & $4,9 \mathrm{Ba}$ \\
\hline 0,75 & $3,0 \mathrm{Ba}$ & $1,4 \mathrm{Aa}$ & $17,7 \mathrm{Ba}$ & $9,4 \mathrm{Aa}$ & $1,9 \mathrm{Bb}$ & $1,0 \mathrm{Bb}$ & $18,0 \mathrm{Aa}$ & $4,7 \mathrm{Bb}$ \\
\hline 1,50 & $4,2 \mathrm{Aa}$ & $1,3 \mathrm{Aa}$ & $26,0 \mathrm{Aa}$ & $10,1 \mathrm{Aa}$ & $2,3 \mathrm{Bb}$ & $1,0 \mathrm{Bb}$ & $16,2 \mathrm{Ab}$ & $7,3 \mathrm{Ab}$ \\
\hline
\end{tabular}

${ }^{(1)}$ Letras maiúsculas comparam as médias relativas aos níveis de Al, em cada variável; letras minúsculas, nas linhas, comparam as duas cultivares, em cada nível de alumínio. 
ram sempre mais elevados na cultivar tolerante do que na cultivar sensível, independentemente da concentração de $\mathrm{Al}$ na solução nutritiva. Na presença de Al, o teor de P na cultivar tolerante sofreu redução apenas na concentração de 1,50 mM de Al, enquanto na cultivar sensível a redução foi significativa a partir de $0,75 \mathrm{mM}$ de alumínio. Na cultivar tolerante não foi observada redução no teor de $\mathrm{K}$, na presença de alumínio. Ao contrário, observou-se até mesmo pequeno aumento (9\%) quando as plantas foram submetidas a $0,75 \mathrm{mM}$ de alumínio. Aumentos como este não são incomuns e foram constatados em outras cultivares de arroz submetidas a concentrações consideradas baixas $(370 \mu \mathrm{M})$, porém reduções drásticas ocorrem em concentrações elevadas de Al (2,22 mM) (Fageria \& Carvalho, 1982). Na cultivar sensível houve redução no teor de K, mas apenas na concentração de 1,50 mM de alumínio. O teor de $\mathrm{Ca}$ na cultivar tolerante sofreu redução apenas na concentração mais elevada de Al, enquanto na cultivar sensível ela ocorreu a partir da concentração de $0,75 \mathrm{mM}$, mas não se intensificou na concentração mais elevada de alumínio. A cultivar tolerante apresentou teores de $\mathrm{Ca}$ mais elevados que a cultivar sensível, exceto na concentração mais elevada de $\mathrm{Al}$, no qual não se observou diferença entre elas. O Mg foi, entre os minerais estudados, o que sofreu os maiores decréscimos porcentuais na presença de Al, nas duas cultivares. Assim, na concentração mais elevada de Al, a cultivar tolerante sofreu uma redução no teor de $\mathrm{Mg}$ de $54 \%$, e a sensível, de $71 \%$. Os macronutrientes que mais sofreram os efeitos tóxicos do Al na parte aérea foram, pela ordem: $\mathrm{Mg}>\mathrm{Ca}>\mathrm{P}$ na tolerante e $\mathrm{Mg}>\mathrm{Ca}>\mathrm{P}>\mathrm{K}$ na sensível.

$\mathrm{O} \mathrm{Al}$, entretanto, não apenas influenciou nos teores de $\mathrm{P}, \mathrm{K}, \mathrm{Ca}$ e $\mathrm{Mg}$, como também reduziu as taxas de absorção líquida de todos eles nas duas cultivares (Tabela 1). As taxas de absorção líquida de todos os minerais foram sempre maiores na cultivar tolerante, independentemente da concentração de Al, exceto a de $\mathrm{Mg}$ em plantas tratadas com $0,75 \mathrm{mM}$ de Al, em que não se observou diferença significativa entre as cultivares. O Ca e o $\mathrm{Mg}$ foram os nutrientes que sofreram as maiores reduções porcentuais em suas taxas de absorção líquida, especialmente na cultivar sensível. Em outras cultivares de arroz, a taxa de absorção líquida de K não foi afetada pelo Al na cultivar sensível, embora na cultivar tolerante tenha sido observado até mesmo um aumento na taxa de absorção líquida desse nutriente (Jan \& Pettersson, 1995). A diferença na resposta obtida por esses autores em relação ao presente trabalho parece ser resultante da menor concentração de $\mathrm{Al}$ $(0,14 \mathrm{mM})$ utilizada. As taxas de absorção líquida de $\mathrm{P}$ e $\mathrm{Ca}$, encontradas por aqueles autores, reduziram na cultivar sensível, mas não foram afetadas na cultivar tolerante, já a taxa de absorção líquida de $\mathrm{Mg}$ foi a mais reduzida, independentemente da cultivar, conforme também verificado no presente trabalho.

O Al, em níveis elevados, portanto, compete com os cátions por sítios de absorção nos transportadores ou em canais de influxo de cátions monovalentes e divalentes (Rengel \& Robinson, 1989c; Huang et al., 1992; Rengel \& Elliot, 1992; Gassmann \& Schroeder, 1994; Kochian, 1995), reduz sua translocação para a parte aérea ou sítios de utilização (Sivaguru \& Paliwal, 1993; Malkanthi et al., 1995a) ou reduz o suprimento de energia para os sistemas de transporte dependentes de energia (Kochian, 1995). Conseqüentemente promove redução nos teores de quase todos os elementos minerais nas duas partes das plantas, conforme se verificou no presente trabalho. Resultados semelhantes foram obtidos em outras espécies vegetais (Calbo \& Cambraia, 1980; Fageria, 1985; Gassmann \& Schroeder, 1994; Malkanthi et al., 1995b).

$\mathrm{Na}$ ausência de $\mathrm{Al}$, as duas cultivares de arroz não diferiram entre si quanto ao índice de eficiência de utilização de P, K, Ca e Mg (Tabela 1). Nas plantas da cultivar tolerante, o tratamento com $\mathrm{Al}$ aumentou os índices de utilização de $\mathrm{P}, \mathrm{Ca}$ e $\mathrm{Mg}$, principalmente na concentração mais elevada de alumínio. Na cultivar sensível, entretanto, esses índices decresceram em relação ao $\mathrm{P}$ e ao $\mathrm{K}$ e aumentaram em relação ao $\mathrm{Mg}$, mas, neste caso, em intensidade menor do que aconteceu na cultivar tolerante.

A cultivar tolerante, portanto, não apenas foi capaz de absorver em taxas mais elevadas $\mathrm{P}, \mathrm{K}, \mathrm{Ca}$ e $\mathrm{Mg}$, mas também foi capaz de mantê-los em concentrações mais elevadas e, principalmente, utilizá-los mais eficientemente na produção de biomassa do que a cultivar sensível. 
As variáveis estudadas neste trabalho relacionaram-se positivamente com a tolerância ao alumínio. Em arroz, pelo menos nas cultivares estudadas (Fageria \& Carvalho, 1982; Fageria et al., 1988), a tolerância ao $\mathrm{Al}$ parece não se restringir a uma variável única, como a manutenção dos teores de $\mathrm{Ca}$ nas raízes ou de $\mathrm{K}$ na parte aérea, verificado na cultivar tolerante. Ela parece ser o somatório de pequenas diferenças quanto à eficiência na absorção, à capacidade de manter níveis adequados e à eficiência de utilização dos macronutrientes, pelo menos aqueles estudados no presente trabalho. Contudo, é possível que o mesmo conceito se aplique a outros macronutrientes e até mesmo micronutrientes (Hai et al., 1989; Rengel \& Robinson, 1989b) nesta e em outras espécies vegetais.

\section{Conclusões}

1. Os macronutrientes cujos teores são mais reduzidos pelo $\mathrm{Al}$ nas raízes são $\mathrm{K}>\mathrm{Mg}>\mathrm{Pe} \mathrm{Ca}>\mathrm{Mg}=\mathrm{K}>\mathrm{P}$ e na parte aérea, $\mathrm{Mg}>\mathrm{Ca}>\mathrm{P}$ e $\mathrm{Mg}>\mathrm{Ca}>\mathrm{P}>\mathrm{K}$ nas cultivares tolerante e sensível, respectivamente.

2. Os macronutrientes que têm suas taxas de absorção líquida mais reduzidas pelo Al são $\mathrm{Mg}>\mathrm{Ca}>\mathrm{P}>\mathrm{K}$ nas duas cultivares.

3. A eficiência na utilização de $\mathrm{P}, \mathrm{Ca}$ e $\mathrm{Mg}$ aumenta na cultivar tolerante e diminui na sensível, com exceção do $\mathrm{Mg}$, que aumenta.

4. Na presença de Al, a cultivar tolerante é mais eficiente na utilização dos macronutrientes estudados do que a sensível.

\section{Agradecimentos}

Ao CNPq, pelas bolsas de produtividade concedidas ao segundo e quarto autores; à Embrapa-CNPAF, pelo fornecimento das sementes.

\section{Referências}

CALBO, A. G.; CAMBRAIA, J. Efeito do alumínio sobre a composição mineral de dois cultivares de sorgo (Sorghum bicolor L. Moench). Revista Ceres, Viçosa, MG, v. 27, p. 369-378, 1980.

CLARK, J. Characterization of phosphatase of intact maize roots. Journal of Agricultural and Food Chemistry, Washington, v. 23, p. 458-460, 1975.
FAGERIA, N. K. Influence of aluminum in nutrient solutions on chemical composition of two rice cultivars at different growth stages. Plant and Soil, Dordrecht, v. 85, p. 423-429, 1985.

FAGERIA, N. K.; CARVALHO, J. R. P. Influence of aluminum in nutrient solutions on chemical composition in upland rice cultivars. Plant and Soil, Dordrecht, v. 69, p. 31-44, 1982.

FAGERIA, N. K.; WRIGHT, R. J.; BALIGAR, V. C. Rice cultivars response to aluminum in nutrient solution. Communications in Soil Science and Plant Analysis, New York, v. 19, p. 1133-1142, 1988.

FERREIRA, R. P.; CRUZ, C. D.; SEDIYAMA, C. S.; FAGERIA, N. K. Identificação de cultivares de arroz tolerantes à toxidez de alumínio pela técnica multivariada. Pesquisa Agropecuária Brasileira, Brasília, v. 30, n. 6, p. 49-54, jun. 1995.

FURLANI, P. R.; FURLANI, A. M. C. Tolerância a alumínio e eficiência a fósforo em milho e arroz: características independentes. Bragantia, Campinas, v. 50, p. 331-340, 1991.

GASSMANN, W.; SCHROEDER, J. I. Inward-rectifying $\mathrm{K}^{+}$channels in root hairs of wheat: a mechanism for aluminum-sensitive low affinity $\mathrm{K}^{+}$uptake and membrane potential control. Plant Physiology, Rockville, v. 105, p. 1399-1408, 1994.

HAI, T. V.; NGA, T. T.; LAUDELOUT, H. Effect of aluminum on the mineral nutrition of rice. Plant and Soil, The Hague, v. 114, p. 173-185, 1989.

HUANG, J. W.; GRUNES, D. L.; KOCHIAN, L. V. Aluminum effects on the kinetics of calcium uptake into cells of the wheat root apex: quantification of calcium fluxes using a calcium-selective vibrating microelectrode. Planta, Berlin, v. 188, p. 414-421, 1992.

JAN, F.; PETTERSSON, S. Aluminum sensitivity of two upland rice cultivars at various levels of nutrient supply. Journal of Plant Nutrition, New York, v. 18, p. 1323-1335, 1995.

KOCHIAN, L. V. Cellular mechanisms of aluminum toxicity and resistance in plants. Annual Review of Plant Physiology and Plant Molecular Biology, Palo Alto, California, v. 46, p. 237-260, 1995.

LINDEMAN, W. Observations on the behavior of phosphate compounds in Clorella at the transition from dark to light. In: UNITED NATIONS INTERNATIONAL CONFERENCE ON THE PEACEFUL USES OF 
ATOMIC ENERGY, 2., 1958, Geneva. Proceedings... Geneva: United Nations, 1958. v. 24, p. 8-15.

MALKANTHI, D. R. R.; MORITSUGU, M.; YOKOYAMA, K. Effects of low $\mathrm{pH}$ and $\mathrm{Al}$ on absorption and translocation of some essential nutrients in excised barley roots. Soil Science and Plant Nutrition, Tokyo, v. 41 , p. $253-262,1995 \mathrm{a}$.

MALKANTHI, D. R. R.; YOKOYAMA, K; YOSHIDA, T.; MORITSUGU, M.; MATSUSHITA, K. Effects of low $\mathrm{pH}$ and $\mathrm{Al}$ on growth and nutrient uptake of several plants. Soil Science and Plant Nutrition, Tokyo, v. 41, p. 161-165, 1995b.

RENGEL, Z.; ELLIOT, D. C. Mechanism of aluminum inhibition of net ${ }^{45} \mathrm{Ca}^{2+}$ uptake by Amaranthus protoplasts. Plant Physiology, Rockville, v. 98, p. 632-638, 1992.

RENGEL, Z.; ROBINSON, D. L. Aluminum effects on growth and macronutrient uptake by annual ryegrass. Agronomy Journal, Madison, v. 81, p. 208-215, 1989a.

RENGEL, Z.; ROBINSON, D. L. Aluminum effects on micronutrient uptake by annual ryegrass. Journal of Plant Nutrition, New York, v. 20, p. 253-269, 1989b.

RENGEL, Z.; ROBINSON, D. L. Competitive $\mathrm{Al}^{3+}$ inhibition of net $\mathrm{Mg}^{2+}$ uptake by intact Lolium multiflorum roots. Plant Physiology, Rockville, v. 91 p. 1407-1413, 1989c.

SIDDIQI, M. Y.; GLASS, A. D. M. Utilization index: a modified approach to the estimation and comparison of nutrient utilization efficiency in plants. Journal of Plant Nutrition, New York, v. 4, p. 289-302, 1981.

SIVAGURU, M.; JAMES, M. R.; ANBUDURAI, P. R.; BALAKUMAR, T. Characterization of differential aluminum tolerance among rice genotypes cultivated in south India. Journal of Plant Nutrition, New York, v. 15, p. 236-246, 1992.

SIVAGURU, M.; PALIWAL, K. Differential aluminum tolerance in some tropical rice cultivars - II: mechanism of aluminum tolerance. Journal of Plant Nutrition, New York, v. 16, p. 1717-1732, 1993.

TAN, K. T.; KELTJENS, W. G.; FINDENEGG, G. R. Aluminum toxicity in sorghum genotypes as influenced by solution acidity. Soil Science and Plant Nutrition, Tokyo, v. 39, p. 291-298, 1993

WELBANK, P. J. The effect of competition with Agropyron repens and of nitrogen and water supply on the nitrogen content of Impatiens parviflora. Annals of Botany, Oxford, v. 26, p. 361-373, 1962. 